Research Article

\title{
Gamma-Glutamyl Transpeptidase to Platelet Ratio: A New Inflammatory Marker Associated with Outcomes after Cardiac Arrest
}

\author{
Yipin Zhao $\mathbb{D}^{1},{ }^{1}$ Zebin Lin, ${ }^{1}$ Yingying Ji, ${ }^{2}$ Huawei Wang, ${ }^{1}$ Li Xiao, ${ }^{1}$ Qingwei Chen, ${ }^{1}$ \\ and Zhiqin $W u{ }^{1}{ }^{1}$ \\ ${ }^{1}$ Department of General Medicine, The Second Affiliated Hospital of Chongqing Medical University, Chongqing, China \\ ${ }^{2}$ Department of Intensive Care Unit, The Second Affiliated Hospital of Zhengzhou University, Zhengzhou 450014, China \\ Correspondence should be addressed to Zhiqin Wu; 302955@hospital.cqmu.edu.cn
}

Received 22 January 2021; Revised 14 May 2021; Accepted 3 August 2021; Published 15 August 2021

Academic Editor: Carlo Cervellati

Copyright (c) 2021 Yipin Zhao et al. This is an open access article distributed under the Creative Commons Attribution License, which permits unrestricted use, distribution, and reproduction in any medium, provided the original work is properly cited.

\begin{abstract}
Introduction. In recent years, gamma-glutamyl transpeptidase to platelet ratio (GPR) has been proposed as a new inflammatory marker. We aimed to evaluate the association between GPR and outcomes after cardiac arrest (CA). Methods. A total of 354 consecutive patients with CA were included in this retrospective study. Patients were divided into three groups according to tertiles of GPR (low, $n=119$; middle, $n=117$; and high, $n=118$ ). To determine the relationship between GPR and prognosis, a logistic regression analysis was performed. The ability of GPR to predict the outcomes was evaluated by receiver operating characteristic (ROC) curve analysis. Two prediction models were established, and the likelihood ratio test (LRT) and the Akaike Information Criterion (AIC) were utilized for model comparison. Results. Among the 354 patients (age 62 [52, 74], 254/354 male) who were finally included in the analysis, those in the high GPR group had poor outcomes. Multivariate logistic regression analysis revealed that GPR was independently associated with the three outcomes, for ICU mortality (odds ratios $(\mathrm{OR})=1.738,95 \%$ confidence interval $(\mathrm{CI}): 1.221-2.474, P=0.002)$, hospital mortality $(\mathrm{OR}=1.676[1.164-2.413], P$ $=0.005)$, and unfavorable neurologic outcomes $(\mathrm{OR}=1.623[1.121-2.351], P=0.010)$. The area under the ROC curve was 0.611 (95\% Cl: $0.558-0.662$ ) for ICU mortality, 0.600 (95\% CI: $0.547-0.651$ ) for hospital mortality, and 0.602 (95\% CI: 0.549 0.653) for unfavorable neurologic outcomes. Further, the LRT analysis showed that compared with the model without GPR, the GPR-combined model had a higher likelihood ratio $\chi^{2}$ score and smaller AIC. Conclusion. GPR, as an inflammatory indicator, was independently associated with outcomes after CA. GPR is helpful in estimating the clinical outcomes of patients with CA.
\end{abstract}

\section{Introduction}

Cardiac arrest (CA) is one of the leading causes of death worldwide. In the United States, there are about 350,000 cases of out-of-hospital cardiac arrest (OHCA) and 200,000 cases of in-hospital cardiac arrest (IHCA) annually [1, 2], with similarly high numbers in Europe. [3]. Despite advances in treatment concepts and strategies, the prognosis of patients with CA remains poor. The overall survival rates to hospital discharge after OHCA and IHCA were both very low, approximately $12 \%$ and $24 \%$, respectively [4-6]. A high proportion of surviving patients present with neurological deficits, often accompanied by disability and high morbidity due to CA-induced hypoxic-anoxic ischemic brain injury [7, 8]. Each CA patient has different clinical conditions and needs different treatment, and multiple clinical parameters influence the clinical outcome of CA [9]. In clinical practice, it is of great significance to find useful biomarkers as simple prognostic indicators to accurately identify the individuals with poor prognosis in the early stage of $\mathrm{CA}$, formulate personalized treatment strategies, and improve the success rate of rescue treatment. The American Heart Association, the European Resuscitation Council, and the European Society of Intensive Care Medicine recommend using a combination of multiple predictors such as clinical examination, blood parameters, electrophysiological measurements, and imaging 
findings to assess prognosis $[1,10,11]$. However, current guidelines and research are more focused on neurological prognosis.

Cardiac arrest is one of the causes of systemic inflammatory response syndrome (SIRS) [12]. SIRS is activated immediately during cardiac arrest and after the return of spontaneous circulation (ROSC) due to the presence of systemic ischemia-reperfusion [13]. It is considered that this systemic inflammatory change is associated with poor clinical prognosis after CA $[14,15]$. Several studies have proposed that the novel inflammatory marker neutrophil-lymphocyte ratio (NLR) and the immature/total granulocyte (I/T-G) ratio is one of the predictors for the prognosis of CA [1619]. Recently, gamma-glutamyl transpeptidase (GGT) to platelet ratio (GPR), which is easy to calculate and obtain, has been proposed as an inflammatory marker [20]. In cardiovascular disease, it has been found that the GPR can serve as an independent predictor of prognosis in coronary heart disease $(\mathrm{CHD})$ patients undergoing percutaneous coronary intervention (PCI), and elevated GPR is associated with increased all-cause mortality and cardiovascular mortality [21]. A prospective study also found that higher GPR levels are associated with a higher risk of mortality in coronary artery disease (CAD) patients during a median follow-up period of 7.56 years [22]. Elevated GGT levels are considered a marker of inflammation and oxidative stress, which is increasingly recognized in cardiovascular diseases [23, 24]. A cohort study showed that GGT was positively associated with the risk of sudden cardiac death in the general male population during a mean follow-up of 22 years [25]. GGT is also associated with acute myocardial ischemia, which is the most common cause of fatal arrhythmia [26]. In systemic inflammation, the disruption of endothelial integrity promotes platelet adhesion and aggregation, resulting in the decrease of platelet count [27]. A reduction in platelet count was reported to be associated with a higher risk of mortality and unfavorable neurologic outcome at 6 months after CA [28]. Since GGT and platelets are involved in both inflammation and CA, we speculate that GPR may be associated with the prognosis after CA. However, so far, there is no relevant research report. Therefore, in the present study, we aimed to explore the associations between GPR and the outcomes after CA.

\section{Materials and Methods}

2.1. Study Population. In this study, the data we used were all obtained from the Dryad digital repository (10.5061/dryad .qv6fp83). Overall, this was a retrospective cohort study conducted from January 2007 to December 2015 at a single center (Erasme Hospital, Brussels, Belgium). The included cases were CA patients treated in the intensive care unit of this hospital. Because of its retrospective nature, there is no need for informed consent. Full information on the study population has been described in detail previously [29]. This study included comatose patients (Glasgow Coma Scale < 9) caused by IHCA or OHCA. Exclusion criteria include deaths within 24 hours of admission and lack of liver function data. All patients with CA and coma received a 24-hour target temperature management (TTM) aimed at a target temperature of
32-34 ${ }^{\circ} \mathrm{C}$. Midazolam and morphine were used as sedative drugs for deep sedation, and cisatracurium was used to control shivering. Repeated transesophageal and/or transthoracic echocardiography was used to assess cardiac function. Postresuscitation management was performed as previously described [30].

2.2. Data Collection. The following basic clinical characteristics data of the patient were collected in detail: demographic data, past chronic diseases, initial heart rhythm, bystander cardiopulmonary resuscitation (CPR), ROSC time, and total adrenaline application dose. After admission, the Acute Physiological and Chronic Health Assessment (APACHE) II score [31] and the Sequential Organ Failure Assessment (SOFA) score [32] were calculated according to the standards to evaluate the severity of the disease at the early stage of admission. The operating standards of laboratory examinations were carried out following local regulations, using the first blood sample taken after ROSC after admission. Laboratory testing indicators included the following: platelet (PLT), gamma-glutamyl transpeptidase (GGT), total bilirubin (TBIL), lactate dehydrogenase (LDH), alkaline phosphatase (ALP), aspartate (AST) and alanine (ALT) transaminases, prothrombin time (PT), fibrinogen, and international normalized ratio (INR). GPR was calculated as the ratio of gamma-glutamyl transpeptidase to platelet. Length of ICU stay, the use of vasoactive drugs, and mechanical ventilation were recorded. Comorbidities include hypertension (HTN), diabetes mellitus, chronic heart failure ( $\mathrm{CHF})$, chronic renal failure (CRF), CAD, neurological disease, liver cirrhosis, chronic obstructive pulmonary disease (COPD)/asthma, long-term use of corticosteroids, or anticoagulants. Record the use of drugs/interventions that may have hepatotoxicity, including quinolone, $\beta$-lactam, antiepileptic, isoniazid, pyrrole, trimethoprim/sulfamethoxazole, acetaminophen, amiodarone, and metronidazole.

2.3. Definitions. Hypertension was defined as systolic blood pressure $\geq 140 \mathrm{mmHg}$ and diastolic blood pressure $\geq 90$ $\mathrm{mmHg}$ (or currently being treated with antihypertensive medications) [33]. DM status was defined by several criteria: the previous history of diabetes mellitus or current use of hypoglycemic agents, fasting plasma glucose $\geq 7.0 \mathrm{mmol} / \mathrm{L}$ $(\geq 126 \mathrm{mg} / \mathrm{dL})$, or hemoglobin A1c $\geq 6.2 \%$ [34]. Acute liver failure was defined as encephalopathy of any degree with a prothrombin time prolongation of approximately 4-6 seconds or an international normalized ratio $\geq 1.5$ [35]. Hypoxic hepatitis was defined as an elevation of AST and/or ALT to more than 20 times the upper limit of the normal range $(\leq 50 \mathrm{IU} / \mathrm{L})$ in the absence of other causes of hepatocellular necrosis [36]. Acute renal failure was diagnosed according to Acute Kidney Injury Network (AKIN) criteria with reference to serum creatinine levels [37]. The shock was defined as SBP $\leq 90 \mathrm{mmHg}$ or the use of dopamine, norepinephrine, and epinephrine for more than 6 hours and the application of an intra-aortic balloon pump.

2.4. Ascertainment of Outcomes. The primary outcome of the study was all-cause mortality during ICU stay (ICU 
TABLE 1: Baseline clinical characteristics.

\begin{tabular}{|c|c|c|c|c|}
\hline Variable & $\begin{array}{c}\text { Low GPR group } \\
(n=119)\end{array}$ & $\begin{array}{l}\text { Middle GPR group } \\
\quad(n=117)\end{array}$ & $\begin{array}{l}\text { High GPR group } \\
\quad(n=118)\end{array}$ & $P$ \\
\hline Mean age (years) & $62(52,74)$ & $62(52,73)$ & $60(51,75)$ & 0.957 \\
\hline Gender (men), $n(\%)$ & $79(66.4)$ & $95(81.2)$ & $80(67.8)$ & 0.021 \\
\hline Weight (kg) & $78(67,85)$ & $79(70,88)$ & $75(65,85)$ & 0.279 \\
\hline \multicolumn{5}{|l|}{ Arrest characteristics } \\
\hline Bystander-witnessed CA, $n(\%)$ & $102(85.7)$ & $99(84.6)$ & $101(85.6)$ & 0.967 \\
\hline Bystander CPR, $n(\%)$ & $75(63.0)$ & $83(70.9)$ & $81(68.6)$ & 0.409 \\
\hline Adrenaline, $n(\%)$ & $103(86.6)$ & $107(91.5)$ & $107(90.7)$ & 0.416 \\
\hline Out of hospital, $n(\%)$ & $74(62.2)$ & $64(54.7)$ & $63(53.8)$ & 0.362 \\
\hline TTM, $n(\%)$ & $108(90.8)$ & $105(89.7)$ & $102(86.4)$ & 0.541 \\
\hline Noncardiac cause, $n(\%)$ & $46(38.7)$ & $43(36.8)$ & $52(44.1)$ & 0.493 \\
\hline Nonshockable rhythm, $n(\%)$ & $64(53.8)$ & $70(59.8)$ & $75(63.6)$ & 0.303 \\
\hline \multicolumn{5}{|l|}{ Corticoids } \\
\hline Chronic heart failure, $n(\%)$ & $23(19.3)$ & $25(21.4)$ & $29(24.6)$ & 0.614 \\
\hline Hypertension, $n(\%)$ & $54(45.4)$ & $47(40.2)$ & $51(43.2)$ & 0.719 \\
\hline Coronary artery disease, $n(\%)$ & $52(43.7)$ & $39(33.3)$ & $48(40.7)$ & 0.246 \\
\hline Diabetes, $n(\%)$ & $27(22.7)$ & $35(29.9)$ & $23(19.5)$ & 0.160 \\
\hline COPD/asthma, $n(\%)$ & $21(17.6)$ & $13(11.1)$ & $25(21.2)$ & 0.110 \\
\hline Neurological disease, $n(\%)$ & $15(12.6)$ & $16(13.7)$ & $20(16.9)$ & 0.612 \\
\hline Chronic renal failure, $n(\%)$ & $18(15.1)$ & $22(18.8)$ & $20(16.9)$ & 0.753 \\
\hline Liver cirrhosis, $n(\%)$ & $1(0.8)$ & $2(1.7)$ & $9(7.6)$ & 0.007 \\
\hline Corticosteroids, $n(\%)$ & $17(14.3)$ & $25(21.4)$ & $36(30.5)$ & 0.010 \\
\hline Chronic anticoagulation, $n(\%)$ & $18(15.1)$ & $16(13.7)$ & $27(22.9)$ & 0.132 \\
\hline \multicolumn{5}{|l|}{ During ICU stay } \\
\hline IABP, $n(\%)$ & $7(5.9)$ & $6(5.1)$ & $11(9.3)$ & 0.394 \\
\hline $\mathrm{ECMO}, n(\%)$ & $11(9.2)$ & $18(15.4)$ & $16(13.6)$ & 0.347 \\
\hline Shock, $n(\%)$ & $50(42.0)$ & $60(51.3)$ & $80(67.8)$ & $<0.001$ \\
\hline Vasopressor therapy, $n(\%)$ & $80(67.2)$ & $85(72.6)$ & $103(87.3)$ & 0.001 \\
\hline Inotropic agents, $n(\%)$ & $51(42.9)$ & $64(54.7)$ & $78(66.1)$ & 0.002 \\
\hline Mechanical ventilation, $n(\%)$ & $117(98.3)$ & $116(99.1)$ & $116(98.3)$ & 0.823 \\
\hline CRRT, $n(\%)$ & $15(12.6)$ & $17(14.5)$ & $25(21.2)$ & 0.170 \\
\hline Paracetamol, $n(\%)$ & $71(59.7)$ & $64(54.7)$ & $55(46.6)$ & 0.127 \\
\hline Amiodarone, $n(\%)$ & $58(48.7)$ & $60(51.3)$ & $57(48.3)$ & 0.886 \\
\hline$\beta$-Lactams, $n(\%)$ & $49(41.2)$ & $46(39.3)$ & $52(44.1)$ & 0.758 \\
\hline Quinolones, $n(\%)$ & $3(2.5)$ & $3(2.6)$ & $0(0.0)$ & 0.217 \\
\hline Azoles, $n(\%)$ & $2(1.7)$ & $3(2.6)$ & $3(2.5)$ & 0.873 \\
\hline Isoniazid, $n(\%)$ & - & - & - & NA \\
\hline TMP/SMX, $n(\%)$ & - & - & - & NA \\
\hline Metronidazole, $n(\%)$ & $2(1.7)$ & - & - & 0.137 \\
\hline Chemotherapy, $n(\%)$ & - & - & - & NA \\
\hline AKI, $n(\%)$ & $63(52.9)$ & $72(61.5)$ & $75(63.6)$ & 0.210 \\
\hline $\mathrm{HH}, n(\%)$ & $6(5.0)$ & $8(6.8)$ & $12(10.2)$ & 0.308 \\
\hline ALF, $n(\%)$ & $54(45.4)$ & $66(56.4)$ & $77(65.3)$ & 0.009 \\
\hline ICU length of stay (days) & $5(3,8)$ & $4(2,11)$ & $4(2,9)$ & 0.852 \\
\hline APACHE II score & $25(21,29)$ & $23(19,28)$ & $24(19,30)$ & 0.218 \\
\hline SOFA score & $11(9,14)$ & $11(9,13)$ & $11(9,14)$ & 0.499 \\
\hline Lowest $\mathrm{ScvO}_{2} / \mathrm{SvO}_{2}(\%)$ & $62.3(57.7,66.0)$ & $62.0(56.8,67.0)$ & $62.7(54.0,67.0)$ & 0.948 \\
\hline Lowest platelet count $\left(\mathrm{mm}^{3}\right)$ & $166(129,216)$ & $138(93,192)$ & $89(56,129)$ & $<0.001$ \\
\hline Laboratory findings on admission & & & & \\
\hline
\end{tabular}


TABLE 1: Continued.

\begin{tabular}{|c|c|c|c|c|}
\hline Variable & $\begin{array}{c}\text { Low GPR group } \\
(n=119)\end{array}$ & $\begin{array}{l}\text { Middle GPR group } \\
\quad(n=117)\end{array}$ & $\begin{array}{l}\text { High GPR group } \\
\quad(n=118)\end{array}$ & $P$ \\
\hline Lactate $\left(\mathrm{m} \mathrm{Eql}^{-1}\right)$ & $4.8(3.8,7.3)$ & $5.10(4.3,7.4)$ & $5.3(4.1,8.0)$ & 0.319 \\
\hline $\mathrm{CRP}\left(\mathrm{mg} \mathrm{dL}^{-1}\right)$ & $31(10,66)$ & $40(14,90)$ & $44(18,98)$ & 0.200 \\
\hline Creatinine $\left(\mathrm{mg} \mathrm{dL}^{-1}\right)$ & $1.2(0.9,1.6)$ & $1.3(1.0,1.6)$ & $1.2(0.9,1.6)$ & 0.595 \\
\hline $\mathrm{ScvO}_{2} / \mathrm{SvO}_{2}(\%)$ & $70.02 \pm(8.90)$ & $69.00 \pm(8.60)$ & $69.13 \pm(10.31)$ & 0.657 \\
\hline AST (IU/L) & $65(36,169)$ & $100(51,186)$ & $115(70,239)$ & 0.001 \\
\hline ALT (IU/L) & $55(28,131)$ & $72(32,142)$ & $78(39,170)$ & 0.062 \\
\hline LDH (IU/L) & $295(216,425)$ & $352(249,524)$ & $372(253,534)$ & 0.010 \\
\hline ALP (IU/L) & $66(52,88)$ & $70(60,102)$ & $89(64,128)$ & $<0.001$ \\
\hline GGT (IU/L) & $37(22,55)$ & $73(55,88)$ & $115(82,196)$ & $<0.001$ \\
\hline Total bilirubin $\left(\mathrm{mg} \mathrm{dL}^{-1}\right)$ & $0.43(0.29,0.64)$ & $0.50(0.36,0.85)$ & $0.61(0.39,1.20)$ & $<0.001$ \\
\hline APTT (sec) & $31.8(26.1,41.3)$ & $32.1(27.7,46.0)$ & $34.70(28.4,45.4)$ & 0.168 \\
\hline PT (\%) & $69.28 \pm(21.74)$ & $59.25 \pm(22.39)$ & $63.31 \pm(23.02)$ & 0.002 \\
\hline INR & $1.20(1.08,1.42)$ & $1.27(1.13,1.53)$ & $1.35(1.20,1.70)$ & 0.001 \\
\hline Platelets $\left(\mathrm{mm}^{3}\right)$ & $238(188,322)$ & $211(158,249)$ & $137(84.25,190)$ & $<0.001$ \\
\hline Proteins $\left(\mathrm{mg} \mathrm{dL}^{-1}\right)$ & $5.6(5.1,6.5)$ & $5.8(5.0,6.3)$ & $5.7(5.0,6.2)$ & 0.872 \\
\hline Glucose $\left(\mathrm{mg} \mathrm{dL}^{-1}\right)$ & $211(169,301)$ & $210(150,297)$ & $190(147,264)$ & 0.132 \\
\hline $\mathrm{pH}$ & $7.29(7.22,7.38)$ & $7.31(7.23,7.38)$ & $7.29(7.19,7.36)$ & 0.552 \\
\hline $\mathrm{PaCO}_{2}(\mathrm{mmHg})$ & $123(86,186)$ & $111(91,162)$ & $109(83,177)$ & 0.510 \\
\hline $\mathrm{PaO}_{2}(\mathrm{mmHg})$ & $38(33,45)$ & $37(32,43)$ & $37(32,43)$ & 0.632 \\
\hline MAP (mmHg) & $91(76,108)$ & $90(77,104)$ & $84(75,99)$ & 0.093 \\
\hline
\end{tabular}

Abbreviation: ICU: intensive care unit; CA: cardiac Arrest; CPR: cardiopulmonary resuscitation; ROSC: return of spontaneous circulation; TTM: targeted temperature management; COPD: chronic obstructive pulmonary disease; IABP: intra-aortic balloon pump; ECMO: extracorporeal membrane oxygenation;

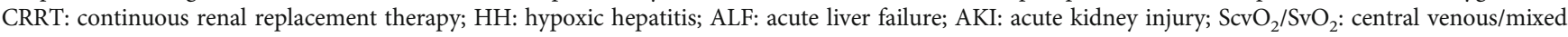
venous oxygen saturation; AST: aspartate aminotransferase; ALT: alanine aminotransferase; LDH: lactate dehydrogenase; ALP: alkaline phosphatase; GGT: $\gamma$-glutamyl transferase; APTT: activated partial thromboplastin time; PT: prothrombin time; INR: international normalized ratio; MAP: mean arterial pressure; CRP: C-reactive protein; APACHE: Acute Physiology and Chronic Health Evaluation; SOFA: Sequential Organ Failure Assessment.

mortality). In-hospital mortality as well as the poor neurological functional outcome at 3 months after CA were defined as secondary endpoints. Assessment of neurological function was performed at 3 months after cardiac arrest by the cerebral performance category (CPC) score during follow-up (1, no or mild neurological dysfunction; 2, moderate neurological dysfunction; 3, severe neurological dysfunction; 4, vegetative state; and 5, death) [38]. Based on the scoring results, CPC 3-5 indicate a poor neurological outcome and CPC 12 are considered a good neurological outcome [39].

2.5. Statistical Analysis. Statistical analysis was performed using SPSS 26 (IBM SPSS, Armonk, New York, NY, USA), MedCalc 19.6.0 (MedCalc Software, Mariakerke, Belgium) and R 4.0.3 software (R Foundation for Statistical Computing, Vienna, Austria). A two-tailed $P$ value of $<0.05$ was defined as statistically significant. For all continuous variables, the Kolmogorov-Smirnov test was used to assess whether they were normally distributed. Data with normal distribution were presented as means $\pm \mathrm{SD}$, and one-way analysis of variance was applied to determine statistical differences among groups. For those data that were not normally distributed, we expressed it using the median (lower quartile, upper quartile), and the comparison of differences among the groups was performed using the Kruskal-Wallis test. We present categorical variables as percentages, and comparisons were made using the chi-square test or the Fisher exact test. We established logistic regression models to assess the effect of GPR on three outcomes. We corrected for age, male gender, $\mathrm{CHF}, \mathrm{CRF}$, adrenaline, lowest $\mathrm{ScvO}_{2}$, lactate value at admission to ICU, bystander-witnessed CA, bystander $\mathrm{CPR}, \mathrm{ScvO}_{2} / \mathrm{SvO}_{2}$ at admission, AST, ALT, LDH, and PAL as potential confounders. Receiver operating characteristic (ROC) curves were plotted. The predictive validity of GPR on outcomes was evaluated by the area under the curve (AUC). Likelihood ratio test (LRT) and the Akaike Information Criterion (AIC) were utilized for model comparison.

\section{Results}

3.1. Baseline Characteristics. Of the total $435 \mathrm{CA}$ patients, there were 51 early deaths and another 30 patients who lacked the necessary information were excluded. Finally, 354 patients were included in the analysis. There were divided into three groups according to tertiles of calculated GPR values (low GPR group, $<0.245, n=119$; middle GPR group, 0.245-0.486, $n=117$; high GPR group, $>0.486, n=$ 118). Baseline clinical characteristics of the present study population are shown in Table 1 . There was no significant 
difference between the three groups in terms of demography and corticoids, except that the proportion of men in the middle GPR group was higher and in the high GPR group had more patients with liver cirrhosis and corticosteroid use (Table 1). During ICU stay, patients in the high GPR group had more frequently developed shock and acute liver failure, were more likely to receive vasopressin and inotropic drugs, and had a lower minimum platelet count(Table 1). For the laboratory findings on admission, there were significant differences among the three groups in AST, LDH, ALP, GGT, total bilirubin, PT, INR, and PLT (all $P<0.05$ ) (Table 1). In Pearson correlation analysis, significant correlations were found between GPR and lactate $(r=0.203, P<0.001)$, ALP $(r=0.381, P<0.001)$, and total bilirubin $(r=0.161, P=$ 0.002 ) (Table 2).

3.2. Clinical Outcomes. Of the 354 patients, 184 (52\%) patients died during their ICU stay, of which there was higher ICU mortality in the high GPR group (65.3\%). 201 (56.8\%) patients died during hospitalization, with the highest rate of hospital mortality in the high GPR group (69.5\%). 141 (39.8\%) patients presented an unfavorable neurologic outcome, with the highest rate in the high GPR group (72.0\%) (Table 3). We analyzed the relationship of GPR as a hierarchical variable with mortality and unfavorable neurologic outcomes, with the low GPR group as the reference. In univariate analysis, high GPR was all associated with the three outcomes (Table 4). This relationship remained significant in multivariate logistic regression analysis for the three outcomes (Table 4). In multivariate logistic regression analysis with GPR as a continuous variable, GPR was still associated with the three outcomes (Table 5). The ROC curve results showed that the AUC was 0.611 (95\% Cl: 0.558-0.662) for ICU mortality, 0.600 (95\% Cl: $0.547-0.651$ ) for hospital mortality, and 0.602 (95\% Cl: 0.549-0.653) for unfavorable neurologic outcomes (Figure 1).

3.3. Adding the GPR to Clinical Information. As shown in Table 6, we built two multivariate models and evaluated the models using likelihood ratio (LR) $\chi^{2}$ values, Akaike Information Criterion (AIC), and AUROC. For the three clinical outcomes, the likelihood ratio (LR) $\chi^{2}$ values for model 2 were all larger than those for model 1. For ICU mortality, the AIC values in models 1 and 2 were 441.471 and 427.015, respectively, and the AUROC increased from 0.734 in model 1 to 0.761 in model 2 . For hospital mortality, the AIC values were 444.029 and 431.416 in model 1 and model 2, respectively, and the AUROC increased from 0.721 in model 1 to 0.749 in model 2. For the unfavorable neurologic outcome, the AIC values were 444.160 and 433.726 in model 1 and model 2, respectively, and the AUROC increased from 0.706 in model 1 to 0.733 in model 2. These all suggested that combining GPR with model 1 could be a better prognostic prediction model.

\section{Discussion}

In this study, we explored for the first time the relationship between GPR and prognosis after CA. Our results indicated
TABLE 2: Correlation analysis

\begin{tabular}{lcc}
\hline Variable & $r$ value & $P$ value \\
\hline Lowest $\mathrm{ScvO}_{2} / \mathrm{SvO}_{2}(\%)$ & -0.046 & 0.387 \\
Lowest platelet count $\left(\mathrm{mm}^{3}\right)$ & -0.226 & $<0.001$ \\
Lactate $\left(\mathrm{mEql}^{-1}\right)$ & 0.203 & $<0.001$ \\
$\mathrm{CRP}\left(\mathrm{mg} \mathrm{dL}^{-1}\right)$ & 0.075 & 0.157 \\
Creatinine $\left(\mathrm{mg} \mathrm{dL}^{-1}\right)$ & 0.010 & 0.854 \\
$\mathrm{Glucose}\left(\mathrm{mg} \mathrm{dL}^{-1}\right)$ & -0.055 & 0.301 \\
$\mathrm{ScvO}_{2} / \mathrm{SvO}_{2}(\%)$ & -0.039 & 0.469 \\
$\mathrm{AST}(\mathrm{IU} / \mathrm{L})$ & 0.104 & 0.050 \\
$\mathrm{ALT}(\mathrm{IU} / \mathrm{L})$ & 0.093 & 0.081 \\
$\mathrm{LDH}(\mathrm{IU} / \mathrm{L})$ & 0.093 & 0.082 \\
$\mathrm{ALP}(\mathrm{IU} / \mathrm{L})$ & 0.381 & $<0.001$ \\
Total bilirubin $\left(\mathrm{mg} \mathrm{dL}^{-1}\right)$ & 0.161 & 0.002 \\
APTT & $<0.001$ & 0.997 \\
PT $(\%)$ & -0.017 & 0.757 \\
INR & 0.033 & 0.541 \\
\hline
\end{tabular}

Abbreviation: ALP: alkaline phosphatase; ALT: alanine aminotransferase; APTT: activated partial thromboplastin time; AST: aspartate aminotransferase; CRP: C-reactive protein; GGT: $\gamma$-glutamyl transferase; INR: international normalized ratio; LDH: lactate dehydrogenase; PLT: platelets; PT: prothrombin time; $\mathrm{ScvO}_{2} / \mathrm{SvO}_{2}$ : central venous/mixed venous oxygen saturation.

that higher GPR was independently associated with higher ICU mortality and hospital mortality and unfavorable neurologic outcome after CA. And this relationship remained for GPR after adjustment for other confounders. GPR, as a new clinical marker, was able to increase the predictive ability of the original model for prognosis when it was added to the original model. Furthermore, as our study population included patients with IHCA and OHCA, this increases the scope to which the present findings apply.

In recent years, the role of hematological markers in cardiovascular diseases has been increasingly recognized. New inflammatory markers, such as the lactate/albumin ratio, I/T-G ratio, and NLR have been shown to be one of effective biomarkers for predicting the prognosis of CA patients [16$19,40]$. GPR as a new clinical biomarker was first proposed in 2016 to be one of the predictors of liver fibrosis and cirrhosis in patients with chronic hepatitis B virus infection. Subsequently, multiple studies have demonstrated the predictive value of GPR for the prognosis of patients with liver cancer and acute/chronic liver failure [41-44]. The results of a retrospective cohort study indicate that GPR is an independent predictor of adverse outcomes in patients with CHD after PCI [21].

The GPR is easily available and rapidly evaluated and can provide a reference for prognosis at an early stage after CA. In this study, we developed a multivariate model, which could better reflect the relevant information of poor prognosis after CA. GPR as a continuous variable could be considered as one of the independent predictors of ICU mortality (OR: 1.738, $P=0.002$ ), hospital mortality (OR: 1.676, $P=$ 0.005 ), and unfavorable neurologic outcome (OR: 1.623, $P$ $=0.010)$. In addition, there was a significant increase when 
TABle 3: Primary and secondary outcomes.

\begin{tabular}{|c|c|c|c|c|c|}
\hline Outcome & All patients $(n=354)$ & $\begin{array}{l}\text { Low GPR group } \\
\quad(n=119)\end{array}$ & $\begin{array}{l}\text { Middle GPR group } \\
\quad(n=117)\end{array}$ & $\begin{array}{l}\text { High GPR group } \\
\quad(n=118)\end{array}$ & $P$ \\
\hline ICU mortality, $n(\%)$ & $184(52.0)$ & $51(42.9)$ & $56(47.9)$ & $77(65.3)$ & $<0.001$ \\
\hline Hospital mortality, $n(\%)$ & $201(56.8)$ & $59(49.6)$ & $60(51.3)$ & $82(69.5)$ & 0.003 \\
\hline Unfavorable neurologic outcome, $n(\%)$ & $213(60.2)$ & $62(52.1)$ & $66(56.4)$ & $85(72.0)$ & 0.004 \\
\hline
\end{tabular}

Abbreviation: ICU: intensive care unit.

TABLE 4: Logistic regression analysis for the GPR as a hierarchical variable.

\begin{tabular}{|c|c|c|c|c|c|}
\hline Outcome & Baseline GPR & Unadjusted OR (95\% CI) & $P$ & Adjusted OR (95\% CI) & $P$ \\
\hline \multirow[t]{3}{*}{ ICU mortality } & Low & Ref. & & Ref. & \\
\hline & Middle & $1.224(0.733,2.045)$ & 0.440 & $1.149(0.644,2.050)$ & 0.639 \\
\hline & High & $2.504(1.482,4.232)$ & 0.001 & $2.162(1.175,3.981)$ & 0.013 \\
\hline \multirow[t]{3}{*}{ Hospital mortality } & Low & Ref. & & Ref. & \\
\hline & Middle & $1.070(0.643,1.783)$ & 0.794 & $0.952(0.537,1.689)$ & 0.868 \\
\hline & High & $2.316(1.361,3.942)$ & 0.002 & $1.915(1.041,3.524)$ & 0.037 \\
\hline \multirow[t]{3}{*}{ Unfavorable neurologic outcome } & Low & Ref. & & Ref. & \\
\hline & Middle & $1.190(0.713,1.987)$ & 0.507 & $1.061(0.601,1.875)$ & 0.838 \\
\hline & High & $2.368(1.381,4.061)$ & 0.002 & $1.954(1.058,3.610)$ & 0.032 \\
\hline
\end{tabular}

Abbreviation: CI: confidence interval; ICU: intensive care unit; OR: odds ratio.

TABLE 5: Logistic regression analysis for the GPR as a continuous variable.

\begin{tabular}{|c|c|c|c|c|}
\hline Outcome & Unadjusted OR (95\% CI) & $P$ & Adjusted OR (95\% CI) & $P$ \\
\hline ICU mortality & $1.694(1.225,2.342)$ & 0.001 & $1.738(1.221,2.474)$ & 0.002 \\
\hline Hospital mortality & $1.689(1.199,2.377)$ & 0.003 & $1.676(1.164,2.413)$ & 0.005 \\
\hline Unfavorable neurologic outcome & $1.654(1.166,2.345)$ & 0.005 & $1.623(1.121,2.351)$ & 0.010 \\
\hline
\end{tabular}

Abbreviation: CI: confidence interval; ICU: intensive care unit; OR: odds ratio.

combining the GPR with the clinical information in the model (AUC values of 0.761, 0.749, and 0.733, respectively). This suggests that the addition of GPR to clinical information can indeed improve model performance. However, caution is still required for the direct application of GPR into clinical practice.

GGT is an important enzyme involved in the gammaglutamyl cycle in amino acid absorption. The enzyme has a wide distribution in the body and, in addition to its presence in liver tissue, is also found in tissues such as the kidney, pancreas, and heart. It is elevated during decompensation from acute hepatitis, chronic active hepatitis, and cirrhosis. GGT plays a crucial role in the metabolism of glutathione, the most important cellular antioxidant in humans, and elevated levels of GGT are considered a marker of inflammation and oxidative stress $[23,24]$. GGT has also been reported to be involved in ischemia-reperfusion injury [45]. Experimental results have shown that GGT levels rise in an isolated rat heart ische$\mathrm{mia} /$ reperfusion model. In the inflammatory process, GGT upregulates and increases antioxidant capacity and may cause leukotriene-induced inflammation [46]. The relationship between GGT and cardiovascular disease has been revealed in previous studies, and GGT has been confirmed to play a role in the occurrence and development of CVD
[47]. In a meta-analysis including seven studies with a total of 273,141 participants, the results indicated that there was an association between GGT and the occurrence of cardiovascular disease and all-cause mortality [48]. In a prospective cohort study that enrolled 1780 men and followed for up to 22 years, GGT was positively associated with the future risk of sudden cardiac death in the general male population [25]. In our results, the high GGT level in the high GPR group was also associated with a worse prognosis. It is speculated that GGT plays a role in the pathophysiological mechanism after the onset of CA and has an impact on the clinical outcome of patients.

High GPR in our results also corresponded to low platelet counts as well as poor prognosis. In fact, platelet counts are reduced early after CA $[28,49,50]$. In patients after cardiac arrest and resuscitation, ischemia and hypoxia as well as increases in thrombin and catecholamines are considered activators of platelets [51]. Insufficient oxygen supply during and after cardiac arrest can lead to systemic inflammation and vascular endothelial dysfunction, resulting in enhanced vascular permeability and thrombosis [15]. Studies have shown that cardiac arrest causes blood-brain barrier damage, and systemic inflammatory response after cardiac arrest may aggravate cerebral ischemic injury [52]. Gando and Wada 

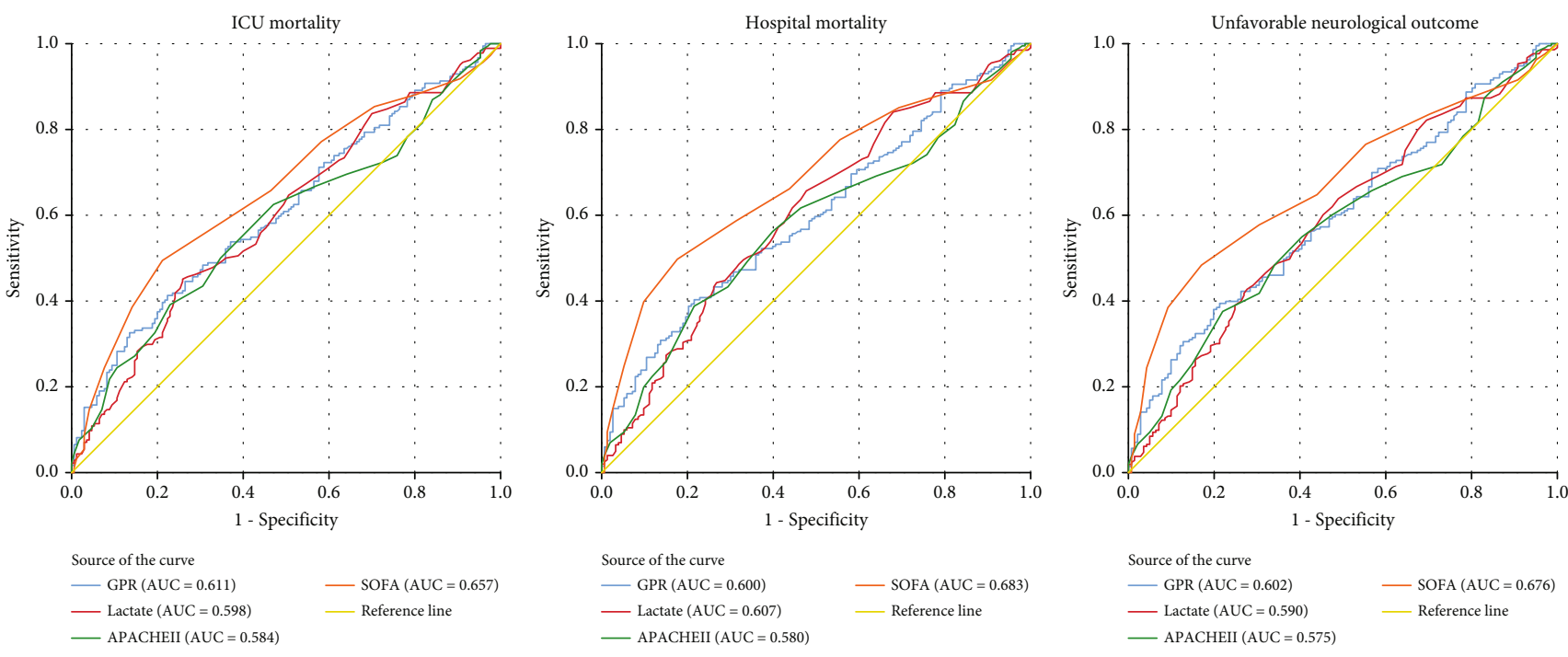

FIGURE 1: ROC curves for predicting ICU mortality, hospital mortality, and unfavorable neurologic outcome.

TABle 6: Comparison of different prognostic models on CA patients.

\begin{tabular}{|c|c|c|c|c|}
\hline Model predictors & Likelihood ratio test $\chi^{2}$ & AIC & $P$ & AUROC \\
\hline \multicolumn{5}{|l|}{ ICU mortality } \\
\hline Model 1 & 64.724 & 441.471 & $<0.001$ & 0.734 \\
\hline Model 2 & 81.179 & 427.015 & $<0.001$ & 0.761 \\
\hline \multicolumn{5}{|l|}{ Hospital mortality } \\
\hline Model 1 & 56.191 & 444.029 & $<0.001$ & 0.721 \\
\hline Model 2 & 70.803 & 431.416 & $<0.001$ & 0.749 \\
\hline \multicolumn{5}{|c|}{ Unfavorable neurologic outcome } \\
\hline Model 1 & 47.842 & 444.160 & $<0.001$ & 0.706 \\
\hline Model 2 & 60.275 & 433.726 & $<0.001$ & 0.733 \\
\hline
\end{tabular}

Abbreviation: AIC: Akaike Information Criterion; AUROC: area under the receiver operating characteristics curve; ICU: intensive care unit. Model 1: age, male gender, witnessed arrest, bystander CPR, adrenaline, lactate, $\mathrm{LDH}$, lowest $\mathrm{ScvO}_{2}$. Model 2: add GPR to model 1.

found that ischemia-reperfusion injury and successful resuscitation caused by CA were associated with endothelial cell activation and were associated with the prognosis of patients with OCHA [51]. Bro-Jeppesen et al. reported that endothelial damage and activation were found within the first $72 \mathrm{~h}$ after CA and that endothelial damage was associated with a high baseline systemic inflammatory level [53]. Systemic ischemia-reperfusion injury after CA causes systemic inflammatory response syndrome (SIRS) [35]. Disseminated intravascular coagulation (DIC) is a common complication of SIRS, which occurs after cardiac arrest and resuscitation [54]. Massive activation of the coagulation pathway along with massive consumption of platelets is the most prominent pathological feature of DIC [55]. Therefore, DIC can lead to extensive microvascular embolization after resuscitation from cardiac arrest [14]. The findings of Kim et al. suggest that an early increased DIC score in OHCA patients is an independent predictor of early mortality and poor longterm prognosis [56]. Prolonged systemic ischemiareperfusion may lead to cerebral and cardiac insufficiency, inadequate tissue oxygen supply, and immune and coagula- tion pathway activation and thus increase the risk of multiple organ failure [57]. Based on the above findings, the relationship between GPR and the prognosis of CA may involve various reasons. GPR is an indicator of the systemic inflammatory response; a reasonable explanation may involve the mechanism of inflammation after CA. However, the exact mechanism and role of GPR need further demonstration.

\section{Limitations}

Our study also has some limitations. First, only the test results of the first blood sample after admission were obtained from the database, without the test results of a certain time interval. Therefore, we cannot judge their causal relationship with clinical outcomes and can only establish statistical links. Second, as a single-center retrospective study, the generalizable application of the findings will be limited. Third, due to the limitation of the research design, some biases are unavoidable, and unknown confounders have the 
potential to influence the results despite adjusting for multiple confounders.

\section{Conclusion}

Elevated GPR on admission was significantly associated with ICU mortality, hospital mortality, and unfavorable neurologic outcome after CA. The predictive value of GPR after CA needs to be confirmed by further multicenter studies.

\section{Data Availability}

All relevant data is available via Dryad: 10.5061/dryad .qv6fp83

\section{Conflicts of Interest}

The authors declare no conflict of interest.

\section{Acknowledgments}

The authors sincerely thank Enrica Iesu et al. for sharing their data. This study was supported by the National Natural Science Foundation of China (Grant No. 31871182).

\section{References}

[1] S. L. Kronick, M. C. Kurz, S. Lin et al., "Part 4: systems of care and continuous quality improvement: 2015 American Heart Association guidelines update for cardiopulmonary resuscitation and emergency cardiovascular care," Circulation, vol. 132, pp. S397-S413, 2015.

[2] R. M. Merchant, L. Yang, L. B. Becker et al., "Incidence of treated cardiac arrest in hospitalized patients in the United States," Critical Care Medicine, vol. 39, no. 11, pp. 24012406, 2011.

[3] B. W. Böttiger and H. K. Van Aken, "Saving 100,000 lives each year in Europe," Best Practice \& Research. Clinical Anaesthesiology, vol. 27, no. 3, pp. 291-292, 2013.

[4] P. S. Chan, B. McNally, F. Tang, A. Kellermann, and Group CS, "Recent trends in survival from out-of-hospital cardiac arrest in the United States," Circulation, vol. 130, no. 21, pp. 1876-1882, 2014.

[5] B. M. Glover, S. P. Brown, L. Morrison et al., "Wide variability in drug use in out-of-hospital cardiac arrest: a report from the resuscitation outcomes consortium," Resuscitation, vol. 83, no. 11, pp. 1324-1330, 2012.

[6] A. Myat, K. J. Song, and T. Rea, "Out-of-hospital cardiac arrest: current concepts," Lancet, vol. 391, no. 10124, pp. 970-979, 2018.

[7] R. G. Geocadin, C. W. Callaway, E. L. Fink et al., "Standards for studies of neurological prognostication in comatose survivors of cardiac arrest: a scientific statement from the American Heart Association," Circulation, vol. 140, no. 9, pp. e517e542, 2019.

[8] T. Cronberg, D. M. Greer, G. Lilja, V. Moulaert, P. Swindell, and A. O. Rossetti, "Brain injury after cardiac arrest: from prognostication of comatose patients to rehabilitation," Lancet Neurology, vol. 19, no. 7, pp. 611-622, 2020.

[9] S. M. Shin, K. S. Kim, G. J. Suh et al., "Prediction of neurological outcomes following the return of spontaneous circulation in patients with out-of-hospital cardiac arrest: retrospective fast-and-frugal tree analysis," Resuscitation, vol. 133, pp. 6570, 2018.

[10] C. W. Callaway, M. W. Donnino, E. L. Fink et al., "Part 8: postcardiac arrest care: 2015 American Heart Association guidelines update for cardiopulmonary resuscitation and emergency cardiovascular care," Circulation, vol. 132, 18 Supplement 2, pp. S465-S482, 2015.

[11] C. Sandroni, A. Cariou, F. Cavallaro et al., "Prognostication in comatose survivors of cardiac arrest: an advisory statement from the European Resuscitation Council and the European Society of Intensive Care Medicine," Resuscitation, vol. 85, no. 12, pp. 1779-1789, 2014.

[12] R. W. Neumar, J. P. Nolan, C. Adrie et al., "Post-cardiac arrest syndrome: epidemiology, pathophysiology, treatment, and prognostication. A consensus statement from the International Liaison Committee on Resuscitation (American Heart Association, Australian and New Zealand Council on Resuscitation, European Resuscitation Council, Heart and Stroke Foundation of Canada, InterAmerican Heart Foundation, Resuscitation Council of Asia, and the Resuscitation Council of Southern Africa); the American Heart Association Emergency Cardiovascular Care Committee; the Council on Cardiovascular Surgery and Anesthesia; the Council on Cardiopulmonary, Perioperative, and Critical Care; the Council on Clinical Cardiology; and the Stroke Council," Circulation, vol. 118, pp. 2452-2483, 2008.

[13] C. Adrie, M. Adib-Conquy, I. Laurent et al., "Successful cardiopulmonary resuscitation after cardiac arrest as a "sepsis-like" syndrome," Circulation, vol. 106, no. 5, pp. 562-568, 2002.

[14] H. Y. Yune, S. P. Chung, Y. S. Park et al., "Delta neutrophil index as a promising prognostic marker in out of hospital cardiac arrest," PLoS One, vol. 10, no. 3, 2015.

[15] J. NBro-Jeppesen, J. Kjaergaard, M. Wanscher et al., "Systemic inflammatory response and potential prognostic implications after out-of-hospital cardiac arrest: A substudy of the target temperature management trial," Critical Care Medicine, vol. 43, no. 6, pp. 1223-1232, 2015.

[16] T. Kong, S. P. Chung, H. S. Lee et al., "The prognostic usefulness of the lactate/albumin ratio for predicting clinical outcomes in out-of-hospital cardiac arrest: a prospective, multicenter observational study (koCARC) study," Shock, vol. 53, no. 4, pp. 442-451, 2020.

[17] V. H. Patel, P. Vendittelli, R. Garg et al., "Neutrophil-lymphocyte ratio: a prognostic tool in patients with in-hospital cardiac arrest," World journal of critical care medicine, vol. 8, no. 2, pp. 9-17, 2019.

[18] H. J. Kim, K. N. Park, S. H. Kim et al., "Association between the neutrophil-to-lymphocyte ratio and neurological outcomes in patients undergoing targeted temperature management after cardiac arrest," Journal of Critical Care, vol. 47, pp. 227-231, 2018.

[19] C. Weiser, M. Schwameis, F. Sterz et al., "Mortality in patients resuscitated from out-of-hospital cardiac arrest based on automated blood cell count and neutrophil lymphocyte ratio at admission," Resuscitation, vol. 116, pp. 49-55, 2017.

[20] M. Lemoine, Y. Shimakawa, S. Nayagam et al., "The gammaglutamyl transpeptidase to platelet ratio (GPR) predicts significant liver fibrosis and cirrhosis in patients with chronic HBV infection in West Africa," Gut, vol. 65, no. 8, pp. 1369-1376, 2016. 
[21] Y. Y. Zheng, T. T. Wu, Y. Chen et al., "Gamma-glutamyl transferase-to-platelet ratio as a novel predictor of long-term adverse outcomes in patients after undergoing percutaneous coronary intervention: a retrospective cohort study," Thrombosis and Haemostasis, vol. 119, no. 6, pp. 1021-1030, 2019.

[22] Q. Chen, Q. Li, D. Li et al., “Association between liver fibrosis scores and the risk of mortality among patients with coronary artery disease," Atherosclerosis, vol. 299, pp. 45-52, 2020.

[23] D. H. Lee, R. Blomhoff, and D. R. Jacobs Jr., "Is serum gamma glutamyltransferase a marker of oxidative stress?," Free Radical Research, vol. 38, no. 6, pp. 535-539, 2004.

[24] E. O. Park, E. J. Bae, B. H. Park, and S. W. Chae, "The associations between liver enzymes and cardiovascular risk factors in adults with mild dyslipidemia," Journal of Clinical Medicine, vol. 9, 2020.

[25] S. K. Kunutsor and H. Khan, " $\gamma$-Glutamyltransferase and risk of sudden cardiac death in middle-aged Finnish men: a new prospective cohort study," Journal of the American Heart Association, vol. 5, no. 2, 2016.

[26] M. J. Davies, "Anatomic features in victims of sudden coronary death. Coronary artery pathology," Circulation, vol. 85, 1 Supplement, pp. I19-I24, 1992.

[27] E. J. Benjamin, S. S. Virani, C. W. Callaway et al., "Heart disease and stroke statistics-2018 update: a report from the American Heart Association," Circulation, vol. 137, no. 12, pp. e67-e492, 2018.

[28] H. J. Kim, K. N. Park, S. H. Kim et al., "Time course of platelet counts in relation to the neurologic outcome in patients undergoing targeted temperature management after cardiac arrest," Resuscitation, vol. 140, pp. 113-119, 2019.

[29] E. Iesu, F. Franchi, F. Zama Cavicchi et al., "Acute liver dysfunction after cardiac arrest," PLoS One, vol. 13, no. 11, 2018.

[30] O. Tujjar, G. Mineo, A. Dell'Anna et al., "Acute kidney injury after cardiac arrest," Critical Care, vol. 19, no. 1, 2015.

[31] W. A. Knaus, E. A. Draper, D. P. Wagner, and J. E. Zimmerman, "APACHE II: a severity of disease classification system," Critical Care Medicine, vol. 13, no. 10, pp. 818-829, 1985.

[32] J. L. Vincent, R. Moreno, J. Takala et al., "The SOFA (Sepsisrelated Organ Failure Assessment) score to describe organ dysfunction/failure," Intensive Care Medicine, vol. 22, no. 7, pp. 707-710, 1996.

[33] C. Lei, B. Wu, M. Liu, and Y. Chen, "Association between hemoglobin $\mathrm{A}_{1 \mathrm{C}}$ levels and clinical outcome in ischemic stroke patients with or without diabetes," Journal of Clinical Neuroscience, vol. 22, no. 3, pp. 498-503, 2015.

[34] A. American Diabetes, "Diagnosis and classification of diabetes mellitus," Diabetes Care, vol. 37, Supplement_1, pp. S81S90, 2014.

[35] D. McDowell Torres, R. D. Stevens, and A. Gurakar, "Acute liver failure: a management challenge for the practicing gastroenterologist," Gastroenterology \& hepatology, vol. 6, no. 7, pp. 444-450, 2010.

[36] J. Henrion, "Hypoxic hepatitis," Liver International, vol. 32, no. 7, pp. 1039-1052, 2012.

[37] R. L. Mehta, J. A. Kellum, S. V. Shah et al., "Acute kidney injury network: report of an initiative to improve outcomes in acute kidney injury," Critical Care, vol. 11, no. 2, p. R31, 2007.

[38] J. P. Nolan, R. A. Berg, L. W. Andersen et al., "Cardiac arrest and cardiopulmonary resuscitation outcome reports: update of the Utstein resuscitation registry template for in-hospital cardiac arrest: a consensus report from a task force of the international Liaison committee on resuscitation (American heart association, European resuscitation Council, Australian and New Zealand Council on resuscitation, heart and stroke foundation of Canada, InterAmerican heart foundation, resuscitation Council of southern africa, resuscitation Council of asia)," Circulation, vol. 140, no. 18, pp. e746-e757, 2019.

[39] B. Jennett and M. Bond, "Assessment of outcome after severe brain damage: a practical scale," Lancet, vol. 1, no. 7905, pp. 480-484, 1975.

[40] B. Sauneuf, C. Bouffard, E. Cornet et al., "Immature/total granulocyte ratio improves early prediction of neurological outcome after out-of-hospital cardiac arrest: the MyeloScore study," Annals of Intensive Care, vol. 6, no. 1, p. 65, 2016.

[41] M. Y. Ke, M. Zhang, Q. Su et al., "Gamma-glutamyl transpeptidase to platelet ratio predicts short-term outcomes in hepatocellular carcinoma patients undergoing minor liver resection," The Journal of Surgical Research, vol. 231, pp. 403-410, 2018.

[42] L. Liu, Q. Lan, L. Lin et al., "Gamma-glutamyl transpeptidaseto-platelet ratio predicts the prognosis in HBV- associated acute-on-chronic liver failure," Clinica Chimica Acta, vol. 476, pp. 92-97, 2018.

[43] D. Luo, H. Li, J. Hu et al., "Development and validation of nomograms based on gamma-glutamyl transpeptidase to platelet ratio for hepatocellular carcinoma patients reveal novel prognostic value and the ratio is negatively correlated with P38MAPK expression," Frontiers in Oncology, vol. 10, p. 548744, 2020.

[44] J. Zhang, T. Wang, L. Xu, P. Wang, M. Zhang, and M. Xu, "Development and validation of a prognostic model based on the albumin-to- fibrinogen ratio (AFR) and gamma-glutamyl transpeptidase-to-platelet ratio (GPR) in hepatocellular carcinoma patients," Clinica Chimica Acta, vol. 511, pp. 107-116, 2020.

[45] M. H. Hanigan, "Chapter three - gamma-glutamyl transpeptidase: redox regulation and drug resistance," in Advances in Cancer Research, D. M. Townsend and K. D. Tew, Eds., vol. Volume 122, pp. 103-141, Academic Press, 2014.

[46] D. Mistry and R. A. Stockley, "Gamma-glutamyl transferase: the silent partner?," COPD, vol. 7, no. 4, pp. 285-290, 2010.

[47] G. Ndrepepa and A. Kastrati, "Gamma-glutamyl transferase and cardiovascular disease," Annals of translational medicine, vol. 4, no. 24, p. 481, 2016.

[48] G. Du, Z. Song, and Q. Zhang, "Gamma-glutamyltransferase is associated with cardiovascular and all-cause mortality: a metaanalysis of prospective cohort studies," Preventive Medicine, vol. 57, no. 1, pp. 31-37, 2013.

[49] A. Cotoia, F. Franchi, C. De Fazio, J. L. Vincent, J. Creteur, and F. S. Taccone, "Platelet indices and outcome after cardiac arrest," BMC Emergency Medicine, vol. 18, no. 1, p. 31, 2018.

[50] A. N. Jeppesen, A.-M. Hvas, A. M. Grejs, C. Duez, S. Ilkjær, and $\mathrm{H}$. Kirkegaard, "Platelet aggregation during targeted temperature management after out-of-hospital cardiac arrest: a randomised clinical trial," Platelets, vol. 29, no. 5, pp. 504$511,2018$.

[51] S. Gando and T. Wada, "Disseminated intravascular coagulation in cardiac arrest and resuscitation," Journal of Thrombosis and Haemostasis, vol. 17, no. 8, pp. 1205-1216, 2019.

[52] J. Bro-Jeppesen, J. Kjaergaard, M. Wanscher et al., "Systemic inflammatory response and potential prognostic implications after out-of-hospital cardiac arrest: a substudy of the target 
temperature management trial," Critical Care Medicine, vol. 43, no. 6, pp. 1223-1232, 2015.

[53] J. Bro-Jeppesen, P. I. Johansson, C. Hassager et al., "Endothelial activation/injury and associations with severity of postcardiac arrest syndrome and mortality after out-of-hospital cardiac arrest," Resuscitation, vol. 107, pp. 71-79, 2016.

[54] M. Levi, "Platelets in critical illness," in Seminars in thrombosis and hemostasis, pp. 252-257, Thieme Medical Publishers, 2016.

[55] F. B. Taylor Jr., C. H. Toh, W. K. Hoots, H. Wada, and M. Levi, "Towards definition, clinical and laboratory criteria, and a scoring system for disseminated intravascular coagulation," Thrombosis and Haemostasis, vol. 86, no. 11, pp. 1327-1330, 2001.

[56] J. Kim, K. Kim, J. H. Lee et al., "Prognostic implication of initial coagulopathy in out-of-hospital cardiac arrest," Resuscitation, vol. 84, no. 1, pp. 48-53, 2013.

[57] S. Gando, T. Kameue, S. Nanzaki, and Y. Nakanishi, "Disseminated intravascular coagulation is a frequent complication of systemic inflammatory response syndrome," Thrombosis and Haemostasis, vol. 75, no. 2, pp. 224-228, 1996. 